\title{
Kostenerstattungen, Geldzahlungen und Sachzuwendungen für die freiwillige Tätigkeit
}

Nadiya Kelle, Nora Karnick \& Laura Romeu Gordo

\section{Kernaussagen}

Bei der Ausübung der freiwilligen Tätigkeit entstehen fast der Hälfte aller Engagierten Kosten. Im Rahmen ihrer freiwilligen Tätigkeit entstehen Männern anteilig häufiger Kosten als Frauen. Anteilig am häufigsten entstehen Personen mit hoher Bildung sowie der Gruppe der 50- bis 64-Jährigen Kosten für ihre freiwillige Tätigkeit.

Eine Kostenerstattung ist für weniger als die Hälfte der Engagierten möglich, denen Kosten bei der Ausübung ihrer freiwilligen Tätigkeit entstehen. Dabei machen nur knapp 16 Prozent dieser Engagierten von einer Kostenausstattung Gebrauch.

2019 erhält ein gutes Fünftel der Engagierten Geldzahlungen sowie Sachzuwendungen für die freiwillige Tätigkeit. Zwischen 1999 und 2009 sind die Anteile an Engagierten, die Geldzahlungen sowie Sachzuwendungen bekommen, etwas gestiegen. Im Trendvergleich zwischen 2009 und 2019 bleiben die Geldzahlungen sowie Sachzuwendungen auf demselben Niveau.

Junge Engagierte erhalten anteilig für ihre freiwillige Tätigkeit im Vergleich zu den anderen Altersgruppen mit Abstand am häufigsten Sachzuwendungen. Die Altersgruppen der 50- bis 64-Jährigen und der 65-Jährigen und Älteren erhalten hingegen anteilig häufiger Geldzahlungen als die jüngeren Altersgruppen. Für ihre freiwillige Tätigkeit erhalten Männer anteilig häufiger Geldzahlungen als Frauen. 
Zwischen den Engagementbereichen zeigen sich große Unterschiede hinsichtlich der Geldzahlungen sowie Sachzuwendungen in der freiwilligen Tätigkeit. Anteilig am häufigsten werden Geldzahlungen und Sachzuwendungen in den Bereichen Justiz und Kriminalitätsprobleme, Unfall- oder Rettungsdienst oder freiwillige Feuerwehr sowie Politik und politische Interessenvertretung getätigt; anteilig am seltensten in den Bereichen Umwelt, Naturschutz oder Tierschutz sowie Schule und Kindergarten. 


\subsection{Einleitung}

Im freiwilligen Engagement können zum einen Zahlungen im Sinne einer Kostenerstattung geleistet werden, die dem Ausgleich erbrachter Auslagen dienen. Zum anderen können Zahlungen in Form materieller oder geldwerter Leistungen (Geldzahlungen beziehungsweise Sachzuwendungen) getätigt werden, die häufig einen $\mathrm{Zu}$ gewinn für Personen bedeuten. Die Geldzahlungen und Sachzuwendungen im freiwilligen Engagement können als Ausdruck der Anerkennung und Wertschätzung für das geleistete Engagement verstanden werden (Vandamme 2018).

Kosten, die für die Ausübung einer freiwilligen Tätigkeit anfallen, können beispielsweise Fahrt- oder Materialkosten sein. Diese Kosten können für einige Bevölkerungsgruppen eine Hürde für den Zugang und die Ausübung des freiwilligen Engagements darstellen. Durch die Kostenerstattung besteht für Engagierte die Möglichkeit, ihre geleisteten Auslagen wieder auszugleichen, sodass Personen möglichst unabhängig von ihren finanziellen Ressourcen ein freiwilliges Engagement ausüben können. Die Kostenerstattung kann also dazu beitragen, die sozialen Ungleichheiten im Zugang zum Engagement und bei der Ausübung des Engagements abzubauen (Romeu Gordo \& Vogel 2017).

Die Sachzuwendungen und Geldzahlungen im freiwilligen Engagement stellen häufig einen geldwerten beziehungsweise monetären Zugewinn für Engagierte dar. Bei Sachzuwendungen kann es sich um Fahrscheine, um eine kostenlose private Nutzung von Räumen oder Ausstattungsmitteln oder um Ehrenamtskarten handeln, die Vergünstigungen beim Eintritt in Museen oder in Schwimmbäder vorsehen können. $\mathrm{Zu}$ den Geldzahlungen können zum einen pauschalierte Aufwandsentschädigungen gezählt werden, die über dem entstandenen Kostenaufwand liegen. Zum anderen kann es sich um eine Steuerbefreiung für Aufwandsentschädigungen handeln, die bei der Ehrenamtspauschale oder den Freibeträgen für Übungsleitende gewährt wird. Weiterhin können die Geldzahlungen für die freiwillige Tätigkeit auch in direkter Form als geringfügige Bezahlungen oder Honorare erfolgen (Jakob 2017).

In der Fachliteratur werden Geldzahlungen, die in direkter Form erfolgen, häufig problematisiert. Zum einen wird angemerkt, dass sich das Engagement in die Nähe entgeltlicher Beschäftigungen verschieben könnte (Jakob 2017; Vandamme 2018). Dabei wird angeführt, dass in einzelnen Bereichen - wie zum Beispiel der Pflege oder der Betreuung demenzkranker Menschen sowie in den Ganztagsschulen bei der Nachmittagsbetreuung - die Finanz- sowie Personalknappheit durch den Einsatz von bezahlten freiwillig Engagierten kompensiert werden könnte. Zum anderen wird argumentiert, dass bei einer Bezahlung im Rahmen der freiwilligen Tätigkeit das Motiv der Einkommenserzielung in den Vordergrund treten könnten (Beyer 2017; Jakob 2017). Bisherige Ergebnisse deuten jedoch auf eine in der Regel geringe Höhe der Zahlungen hin (Romeu Gordo \& Vogel 2017). Außerdem zeigt sich, dass das Motiv, sich durch freiwilliges Engagement etwas dazuverdienen zu wollen, anteilig vergleichsweise selten genannt wird (siehe Kapitel 6). 
In diesem Kapitel wird untersucht, welchen Gruppen von Engagierten die Geldzahlungen und Sachzuwendungen primär zugutekommen: Wie sind die Geldzahlungen und auch Sachzuwendungen über verschiedene Bevölkerungsgruppen verteilt und wie entwickeln sich diese im Zeitvergleich? Außerdem wird untersucht, in welchen gesellschaftlichen Bereichen Zahlungen eingesetzt werden. Dabei kann geklärt werden, ob Zahlungen, wie in der Fachliteratur diskutiert, in erster Linie in gesellschaftlichen Bereichen mit ausgeprägten Handlungsbedarfen geleistet werden. Auch bei den Kostenerstattungen wird untersucht, welchen Bevölkerungsgruppen sie verstärkt zugutekommen. Erhalten die bessergestellten Gruppen in höherem Maße die Möglichkeit zur Kostenerstattung, könnte dies ein Ausdruck sozialer Ungleichheiten im Engagement sein.

Im vorliegenden Kapitel sollen auf Basis der Daten des Freiwilligensurveys folgende Fragen betrachtet werden:

a) Wie unterscheiden sich die Anteile der Engagierten, denen Kosten für ihre freiwillige Tätigkeit entstehen nach Geschlecht, nach Alter und nach Bildung 2019? Wie hoch sind diese Anteile im Zeitvergleich?

b) Für die Engagierten, denen 2019 Kosten für ihre freiwillige Tätigkeit entstehen: Wie hoch sind die Anteile derjenigen, für die eine Kostenerstattung möglich ist (beziehungsweise nicht möglich ist) und die Gebrauch von einer Kostenerstattung machen (beziehungsweise nicht machen)?

c) Wie unterscheiden sich die Anteile der Engagierten, die Geldzahlungen beziehungsweise Sachzuwendungen für ihre freiwillige Tätigkeit erhalten, nach Geschlecht, nach Alter und nach Bildung? Wie hoch sind diese Anteile im Zeitvergleich?

d) Wie unterscheiden sich die Anteile der Engagierten, die Geldzahlungen beziehungsweise Sachzuwendungen für die freiwillige Tätigkeit erhalten, nach gesellschaftlichen Bereichen 2019?

\subsection{Daten und Indikatoren}

Die Ergebnisse des vorliegenden Kapitels beruhen auf den Daten des Deutschen Freiwilligensurveys (FWS) und werden gewichtet dargestellt. Die Gewichtung zielt darauf ab, die Repräsentativität zu verbessern, um verallgemeinerbare Aussagen treffen zu können. In den Veröffentlichungen zu den Erhebungen der Jahre 1999 bis 2014 wurden im Rahmen des Freiwilligensurveys bislang die Gewichtungsmerkmale Geschlecht, Alter, Bundesland sowie Gemeindegrößenklasse herangezogen. Diese Gewichtung wird nun um das Gewichtungsmerkmal der schulischen Bildung ergänzt. Personen aus unterschiedlichen Bildungsgruppen nehmen mit unterschiedlicher Wahrscheinlichkeit an Umfragen teil; daher sind verschiedene Bildungsgruppen in den Daten über- beziehungsweise unterrepräsentiert. Die Gewichtung inklusive 
schulischer Bildung wird in diesem Bericht auf die Daten aller fünf Erhebungswellen von 1999 bis 2019 angewendet. Die Engagementquoten fallen durch dieses Vorgehen für alle Erhebungswellen niedriger aus als bisher berichtet; es ändert sich somit das Niveau, der Trend bleibt jedoch im Wesentlichen bestehen. Die Gewichtung der Daten inklusive Bildung wirkt sich auch auf andere Ergebnisse aus.

Alle Ergebnisse werden auf statistische Signifikanz getestet (verwendet wird ein Signifikanzniveau von $\mathrm{p}<0,05)$. Die Prüfung auf statistische Signifikanz zeigt an, ob ein anhand der Daten des Freiwilligensurveys gefundener Unterschied mit großer Wahrscheinlichkeit auch in der Grundgesamtheit, also beispielsweise in der Wohnbevölkerung Deutschlands, vorhanden ist (statistisch signifikant) oder nur zufällig in den Daten vorliegt (statistisch nicht signifikant).

Die dargestellten Ergebnisse fußen auf der Befragung des Freiwilligensurveys 2019, die von März bis November 2019 erfolgte. Somit ist es mithilfe der Daten des Freiwilligensurveys 2019 nicht möglich, Aussagen über spätere Entwicklungen wie beispielsweise die Auswirkungen der Corona-Pandemie auf das freiwillige Engagement zu tätigen.

In diesem Kapitel werden ausschließlich freiwillig engagierte Personen betrachtet. Die Analysen beziehen sich auf die freiwillige Tätigkeit (einzige Tätigkeit oder zeitaufwendigste Tätigkeit bei mehreren Tätigkeiten). Die Analysen werden im Trend sowie im Vergleich zwischen Frauen und Männern, Altersgruppen und Bildungsgruppen durchgeführt. Diese verwendeten Differenzierungsmerkmale werden in Kapitel 2 beschrieben. Außerdem werden Analysen nach gesellschaftlichen Bereichen durchgeführt, in denen die Engagierten ihre einzige oder zeitaufwendigste Tätigkeit ausüben (zum Engagement in den unterschiedlichen gesellschaftlichen Bereichen siehe Kapitel 5).

Kosten und Kostenerstattungen: Die Entstehung von Kosten bei der freiwilligen Tätigkeit wurde im Freiwilligensurvey 2019 mit der folgenden Frage erfasst:

Entstehen Ihnen Kosten aus Ihrer Tätigkeit, zum Beispiel Fahrt-oder Materialkosten? Mitgliedsbeiträge und Spenden zählen Sie bitte nicht dazu.

Antwortkategorien: ja; nein

Anschließend werden alle Engagierten, die angegeben haben, dass ihnen Kosten aus ihrer Tätigkeit entstehen, gefragt:

Besteht prinzipiell die Möglichkeit für Sie, sich diese Kosten erstatten zu lassen? Antwortkategorien: ja; teilweise; nein

Alle Engagierten, die angegeben haben, dass die Kostenerstattung möglich oder teilweise möglich ist, werden dann gefragt: 


\section{Machen Sie von einer Erstattung der Kosten in der Regel Gebrauch? Antwortkategorien: ja; nein}

Die Abfragen zu den Kosten und Kostenerstattungen sind in den Erhebungsjahren 2014 und 2019 identisch. In den ersten drei Erhebungswellen wurde keine Frage dazu gestellt, ob Engagierten Kosten entstehen. Daher werden für die Analysen im Zeitvergleich Daten aus den Erhebungsjahren 2014 und 2019 verwendet. In die Analysen zu den Kostenerstattungen 2019 werden nur die Engagierten miteinbezogen, die angegeben haben, dass Ihnen Kosten bei ihrer freiwilligen Tätigkeit entstehen.

Geldzahlungen und Sachzuwendungen: Die Erfassung von Geldzahlungen und Sachzuwendungen hat sich über die Zeit geändert. In Tabelle 12-1 werden die Abfragen zu den Geldzahlungen und Sachzuwendungen aus den fünf Erhebungswellen des Freiwilligensurveys dargestellt. Dabei wird ersichtlich, dass die Abfragen in den Jahren 2014 und 2019 identisch waren. Die Geldzahlungen und die Sachzuwendungen wurden anhand von zwei separaten Fragen erfasst. In den Erhebungsjahren 1999 und

Tabelle 12-1 Erfassung der Geldzahlungen und Sachzuwendungen im Zeitvergleich

\begin{tabular}{|c|c|c|}
\hline Welle & Fragetext & $\begin{array}{l}\text { Antwort- } \\
\text { möglichkeiten }\end{array}$ \\
\hline 1999 & $\begin{array}{l}\text { Erhalten Sie persönlich eine gewisse Vergütung, beispielsweise eine pau- } \\
\text { schalierte Aufwandsentschädigung (1), Honorare (2), eine geringfügige } \\
\text { Bezahlung (3), Sachzuwendungen, z. B. Fahrscheine, private Nutzung von } \\
\text { Gruppenräumen oder Ausstattungsmitteln (4), Nein, nichts davon (5). } \\
\text { (Mehrfachnennungen sind möglich) }\end{array}$ & (jeweils) ja; nein \\
\hline
\end{tabular}

2004 Erhalten Sie persönlich eine gewisse Vergütung, beispielsweise eine pau-

(jeweils) ja; nein schalierte Aufwandsentschädigung (1), Honorare (2), eine geringfügige

Bezahlung (3), Nein, nichts davon (4).

(Mehrfachnennungen sind möglich)

Nicht im Zeitvergleich ausgewertet

2009 Erhalten Sie persönlich eine gewisse Vergütung, beispielsweise eine pau-

(jeweils) ja; nein schalierte Aufwandsentschädigung (1), Honorare (2), eine geringfügige Bezahlung (3), Sachzuwendungen, z. B. Fahrscheine, private Nutzung von Gruppenräumen oder Ausstattungsmitteln (4), Nein, nichts davon (5), Keine Angabe (6). (Mehrfachnennungen sind möglich)

2014/ Unabhängig von einer möglichen Kostenerstattung: Haben Sie persön-

2019 lich in den vergangenen 12 Monaten Geld für Ihre freiwillige Tätigkeit erhalten?

Wenn Sie an die letzten 12 Monate denken: Haben Sie im Rahmen Ihrer Tätigkeit Sachzuwendungen erhalten, zum Beispiel Fahrscheine oder eine kostenlose private Nutzung von Räumen oder Ausstattungsmitteln?

ja; nein

ja; nein 
2009 wurden die Geldzahlungen und Sachzuwendungen hingegen zusammen abgefragt. Im Erhebungsjahr 2004 wurden zwar die Geldzahlungen, aber keine Sachzuwendungen abgefragt. Aus diesem Grund wird das Jahr 2004 nicht in die Analyse von Geldzahlungen und Sachzuwendungen im Zeitvergleich aufgenommen.

Für die Analysen im Zeitvergleich werden die Kategorien aus den Erhebungsjahren 1999 und 2009 ,pauschalierte Aufwandsentschädigung', ,Honorare' und ,geringfügige Bezahlungen' zusammengefasst, um den Vergleich zu den Erhebungsjahren 2014 und 2019 zu ermöglichen. Allerdings könnte dadurch die Bedeutung der Geldzahlungen in den ersten Erhebungsjahren überschätzt werden, da pauschalierte Aufwandsentschädigungen keinen finanziellen Zugewinn bedeuten müssen, sondern eine reine Kostenerstattung darstellen können. Dargestellt werden in den Analysen die Anteile der Engagierten, die Geldzahlungen, Sachzuwendungen oder beides erhalten.

\subsection{Kosten und Möglichkeiten der Kostenerstattung}

Im Jahr 2019 entstehen 44,o Prozent der Engagierten Kosten bei der Ausübung ihrer freiwilligen Tätigkeit (Abbildung 12-1a); seit 2014 mit 43,9 Prozent hat sich dieser Anteil nicht verändert. Der Unterschied zwischen den Erhebungsjahren 2014 und 2019 ist nicht statistisch signifikant (Zeitvergleich ohne Abbildung).

Frauen entstehen 2019 mit 41,8 Prozent anteilig seltener Kosten bei der Ausübung ihrer freiwilligen Tätigkeit als Männern mit 46,2 Prozent (Abbildung 12-1a). Die jüngste Altersgruppe der 14- bis 29-Jährigen gibt mit 36,6 Prozent anteilig am seltensten entstehende Kosten für die freiwillige Tätigkeit an, die 50- bis 64-Jährigen mit 49,2 Prozent anteilig am häufigsten. Die Altersgruppen der 30- bis 49-Jährigen sowie der 65-Jährigen und Älteren liegen mit 45,3 Prozent sowie 42,7 Prozent dazwischen.

Engagierte Personen, die noch zur Schule gehen, geben mit 31,9 Prozent im Vergleich aller Bildungsgruppen anteilig am seltensten entstehende Kosten an. Für die übrigen drei Bildungsgruppen ist ein Bildungsgradient zu erkennen. Für ihre freiwillige Tätigkeit entstehen engagierten Personen mit niedriger Bildung anteilig seltener Kosten als engagierten Personen mit mittlerer und hoher Bildung; engagierte Personen mit mittlerer Bildung haben in ihrer freiwilligen Tätigkeit wiederum anteilig seltener Kosten als engagierte Personen mit hoher Bildung (Abbildung 12-1b).

Für diejenigen Engagierten, denen Kosten für ihre freiwillige Tätigkeit entstehen, stellt sich die Frage, ob sie eine Möglichkeit haben, sich diese Kosten erstatten zu lassen. In Abbildung 12-2 werden somit nur die Engagierten betrachtet, die angegeben haben, dass ihnen Kosten bei der Ausübung ihrer freiwilligen Tätigkeit entstehen. Insgesamt zeigt sich, dass für 59, o Prozent der Engagierten, denen Kosten entstehen, keine Kostenerstattung möglich ist (Abbildung 12-2a). Für die weiteren Engagierten, denen Kosten entstehen, ist die Kostenerstattung zwar möglich, dennoch machen lediglich 15,4 Prozent auch Gebrauch davon, 25,6 Prozent nicht. 
Abbildung 12-1 Anteile freiwillig engagierter Personen, denen Kosten für die freiwillige Tätigkeit entstehen, 2019, a) gesamt, nach Geschlecht und nach Alter, b) nach Bildung (Basis: alle Engagierten)

a) gesamt, nach Geschlecht und nach Alter

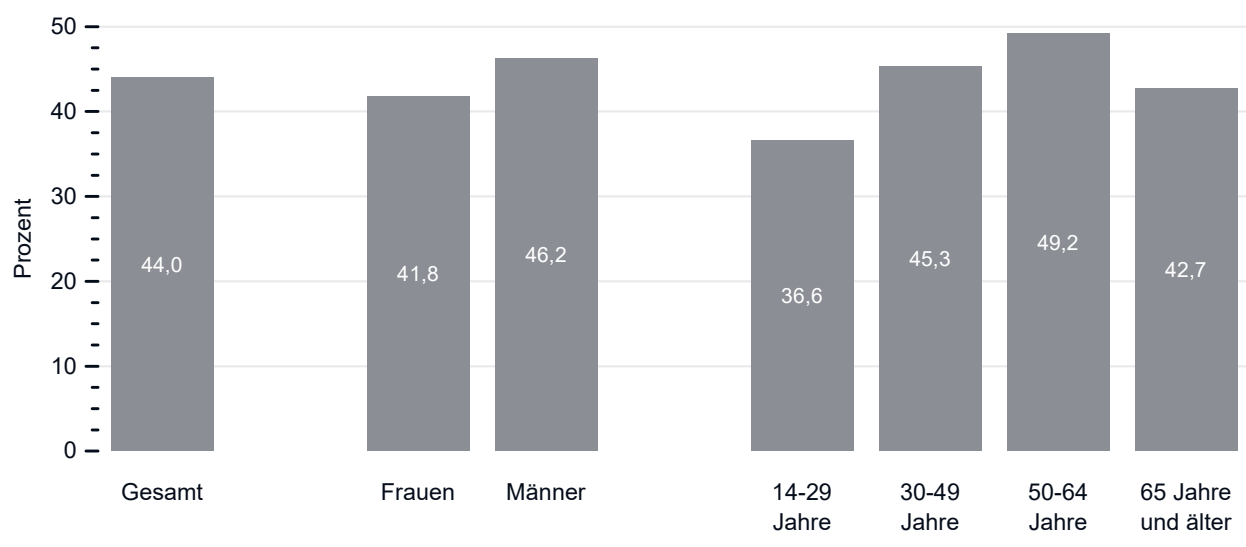

b) nach Bildung

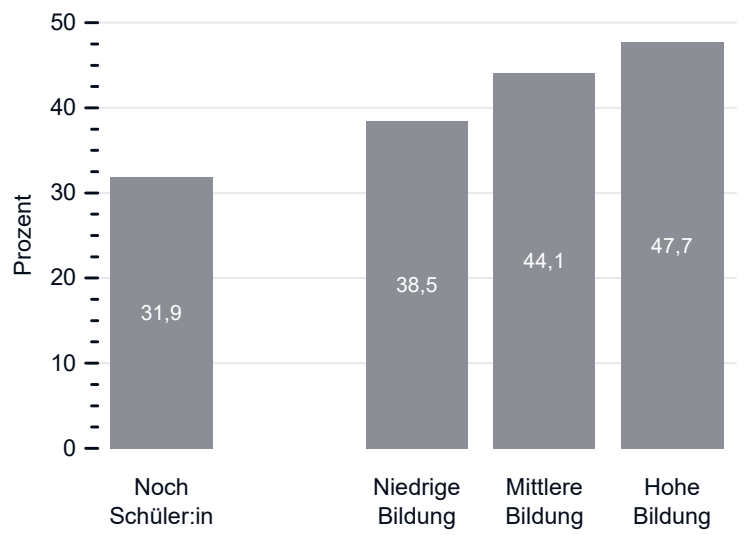

Quelle: FWS 2019, gewichtet, eigene Berechnungen (DZA). Basis: alle Engagierten $(n=11.916)$.

Geschlechterunterschied ist statistisch signifikant.

Altersgruppenunterschiede sind alle statistisch signifikant mit Ausnahme des Unterschieds zwischen den Gruppen der 30- bis 49-Jährigen und 65-Jährigen und Älteren.

Bildungsgruppenunterschiede sind alle statistisch signifikant mit Ausnahme des Unterschieds zwischen den Personen, die noch zur Schule gehen, und den Personen mit niedriger Bildung. 


\section{Abbildung 12-2 Anteile freiwillig engagierter Personen, die Angaben zur Kostenerstattung machen, 2019, a) gesamt, nach Geschlecht und Alter, b) nach Bildung (Basis: alle Engagierten, denen Kosten für die freiwillige Tätigkeit entstehen)}

a) gesamt, nach Geschlecht und nach Alter

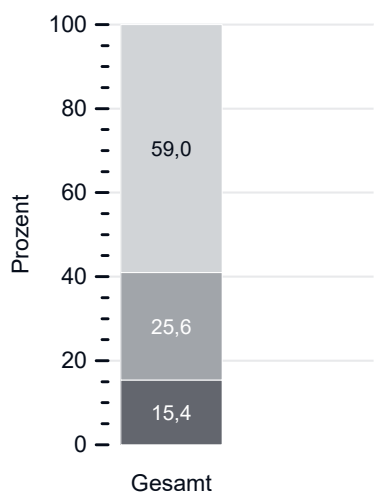

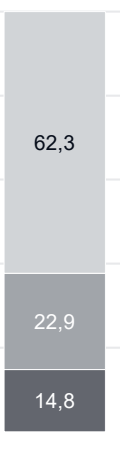

Frauen

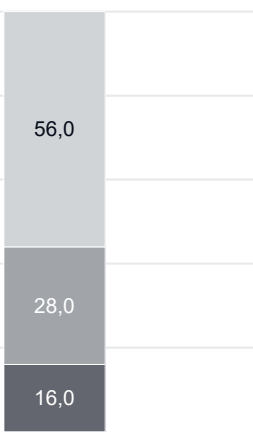

Männer

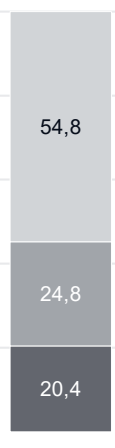

$14-29$

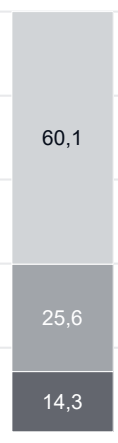

$30-49$

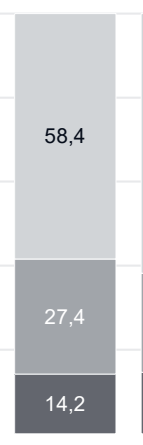

50-64

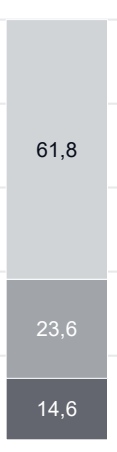

65 Jahre und älter

b) nach Bildung

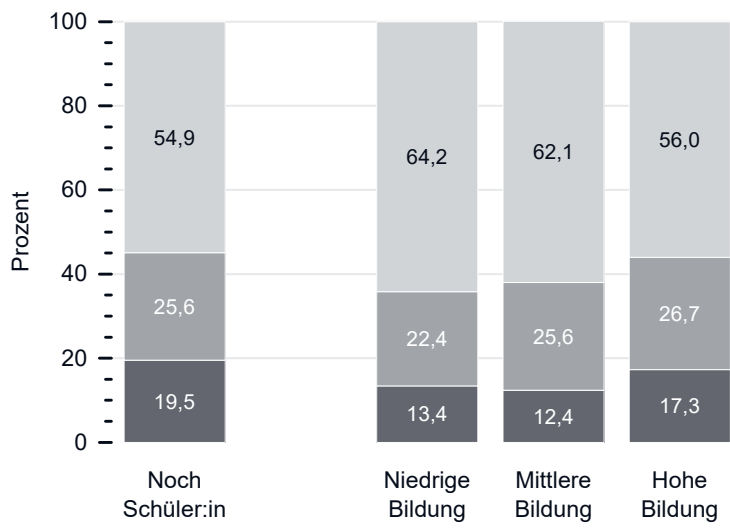

Keine Kostenerstattung möglich

Kostenerstattung möglich und kein Gebrauch

Kostenerstattung möglich und Gebrauch

Quelle: FWS 2019, gewichtet, eigene Berechnungen (DZA). Basis: alle Engagierten, denen Kosten für die freiwillige Tätigkeit entstehen $(\mathrm{n}=5.419)$.

Hinweis: Abweichungen in den Prozentwerten ergeben sich durch das Auf- und Abrunden der Werte.

Für die Kategorie,Keine Kostenerstattung möglich' ist der Geschlechterunterschied statistisch signifikant; Altersgruppenunterschiede sind statistisch signifikant zwischen den 14- bis 29-Jährigen und den 65-Jährigen und Älteren; Bildungsgruppenunterschiede sind statistisch signifikant zwischen den Personen mit hoher Bildung sowohl zu Personen mit niedriger als auch zu Personen mit mittlerer Bildung.

Für die Kategorie,Kostenerstattung möglich und kein Gebrauch' ist der Geschlechterunterschied statistisch signifikant; Altersgruppen- und Bildungsgruppenunterschiede sind nicht statistisch signifikant.

Für die Kategorie, Kostenerstattung möglich und Gebrauch' ist der Geschlechterunterschied nicht statistisch signifikant; Altersgruppenunterschied ist statistisch signifikant zwischen den 14- bis 29-Jährigen und allen anderen Altersgruppen; Bildungsgruppenunterschied ist statistisch signifikant zwischen Personen mit mittlerer Bildung und Personen mit hoher Bildung. 
Diese Anteile unterscheiden sich nach Geschlecht, Altersgruppe und Bildungsgruppe. Engagierte Frauen, denen Kosten entstehen, geben anteilig häufiger als engagierte Männer an, dass für sie keine Kostenerstattung möglich sei (62,3 Prozent zu 56,o Prozent). Somit haben engagierte Frauen anteilig seltener als engagierte Männer die Möglichkeit, sich die für ihre freiwillige Tätigkeit entstandenen Kosten erstatten zu lassen. Frauen und Männer, denen Kosten für ihre freiwillige Tätigkeit entstehen und die eine Möglichkeit der Kostenerstattung haben, machen zu ähnlichen Anteilen davon Gebrauch. Der Unterschied zwischen den entsprechenden Anteilen von 14,8 Prozent bei Frauen und 16,o Prozent bei Männern ist statistisch nicht signifikant.

Die jüngste Altersgruppe der 14- bis 29-Jährigen, die Kosten im Rahmen ihrer freiwilligen Tätigkeit hat, hat anteilig seltener die Möglichkeit zur Kostenerstattung als die älteste Gruppe der 65-Jährigen und Älteren (Abbildung 12-2a). Andere Altersgruppenunterschiede sind für die Möglichkeit der Kostenerstattung nicht statistisch signifikant. Dafür macht die jüngste Altersgruppe mit 20,4 Prozent anteilig am häufigsten Gebrauch von der Kostenerstattung, wenn ihnen Kosten entstehen.

Hinsichtlich der Bildungsgruppenunterschiede zeigt sich, dass Engagierte mit hoher Bildung, denen Kosten entstehen, mit 56,o Prozent anteilig seltener als Engagierte mit niedriger Bildung (64,2 Prozent) und mittlerer Bildung (62,1 Prozent) angeben, dass für sie keine Kostenerstattung möglich sei (Abbildung 12-2b). Mit anderen Worten haben Engagierte mit hoher Bildung anteilig am häufigsten die Möglichkeit zur Kostenerstattung in ihrer freiwilligen Tätigkeit. Zudem machen Engagierte mit hoher Bildung, denen Kosten entstehen, anteilig häufiger von der Möglichkeit zur Kostenerstattung Gebrauch (17,3 Prozent) als Engagierte mit mittlerer Bildung (12,4 Prozent). Zwischen engagierten Personen mit hoher und niedriger Bildung, denen Kosten entstehen, zeigt sich hier allerdings kein statistisch signifikanter Unterschied. Auch für engagierte Personen, die noch zur Schule gehen und denen Kosten im Rahmen der Ausübung ihrer freiwilligen Tätigkeit entstehen, zeigen sich keine statistisch signifikanten Unterschiede zu den anderen Bildungsgruppen. Dies kann auch an der geringen Fallzahl dieser Gruppe liegen $(n=97)$.

\subsection{Geldzahlungen und Sachzuwendungen}

Bei der Betrachtung von Geldzahlungen sowie Sachzuwendungen im Zeitvergleich zeigt sich, dass diese im Jahr 1999 bei 17,5 Prozent lagen und in den Nachfolgejahren etwas höher bei 23,2 Prozent (2009), 22,o Prozent (2014) und 22,2 Prozent (2019). Die Trendunterschiede zwischen den letzten drei Erhebungsjahren sind dabei nicht statistisch signifikant (Abbildung 12-3). Die Anteile an Engagierten, die Sachzuwendungen erhalten, nahmen zwischen 1999 und 2014 zu und bleiben zwischen 2014 und 2019 auf dem gleichen Niveau. Hingegen liegen die Anteile an Engagierten, die Geldzahlungen in den Jahren 2014 und 2019 erhalten haben, auf einem geringeren Niveau als 1999 und 2009. Die Kombination aus Geldzahlungen und Sachzuwendungen kam 1999 mit 
Abbildung 12-3 Anteile freiwillig engagierter Personen, die Geldzahlungen sowie Sachzuwendungen für die freiwillige Tätigkeit erhalten, im Zeitvergleich 1999-2019 (Basis: alle Engagierten)

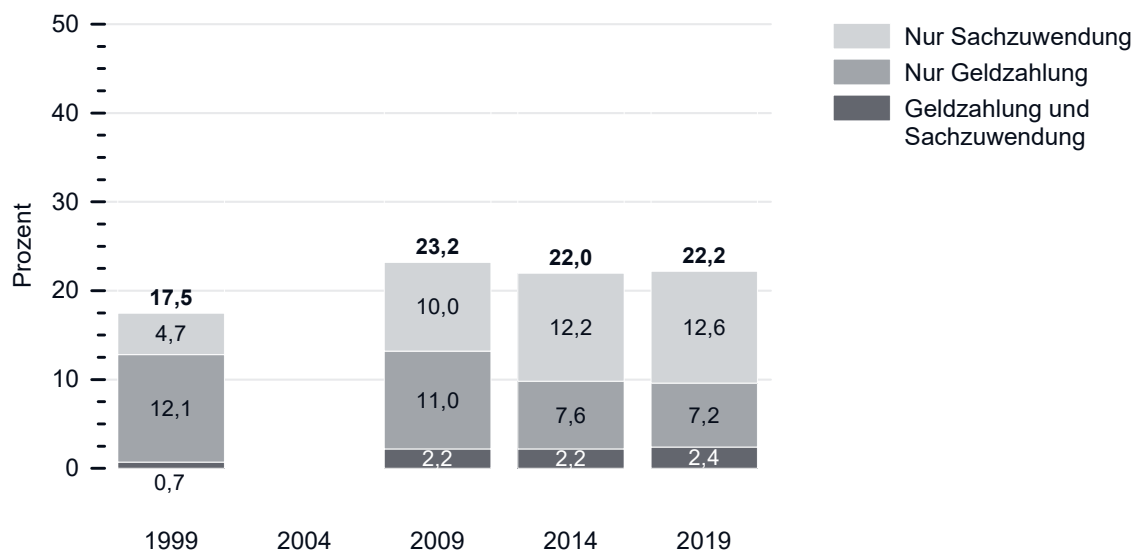

Quelle: FWS, gewichtet, eigene Berechnungen (DZA). Basis: alle Engagierten. FWS 1999 ( $n=4.671)$, FWS 2004 ( $n=5.137)$, FWS 2009 ( $n=7.001$ ), FWS $2014(n=12.408)$, FWS $2019(n=11.899)$

Im Jahr 2004 wurden zwar Geldzahlungen, jedoch keine Sachzuwendungen erhoben. Der Anteil an Geldzahlungen lag 2004 bei 13,4 Prozent.

Für die Gesamtwerte aller Kategorien gibt es statistisch signifikante Trendunterschiede zwischen 1999 zu 2009,1999 zu 2014 und 1999 zu 2019. Für die Kategorie,Nur Sachzuwendung' sind alle Trendunterschiede statistisch signifikant mit Ausnahme des Unterschieds zwischen 2014 und 2019.

Für die Kategorie,Nur Geldzahlung' gibt es statistisch signifikante Trendunterschiede zwischen 1999/2009 zu 2014/2019. Dabei sind die Unterschiede zwischen 1999 und 2009 sowie 2014 und 2019 nicht statistisch signifikant.

Für die Kategorie,Geldzahlung und Sachzuwendung' gibt es statistisch signifikante Trendunterschiede zwischen 1999 zu 2009,1999 zu 2014 und 1999 zu 2019.

o,7 Prozent nur selten vor; in den Nachfolgejahren haben jeweils etwa zwei Prozent der Engagagierten sowohl Sachzuwendungen als auch Geldzahlungen erhalten.

Differenziert nach Geschlecht, Alter und Bildung gibt es deutliche Unterschiede im Hinblick auf den Erhalt von Geldzahlungen, Sachzuwendungen oder beidem im Rahmen der freiwilligen Tätigkeit (Abbildung 12-4a). Engagierte Frauen erhalten mit 20,9 Prozent anteilig seltener Geldzahlungen sowie Sachzuwendungen als engagierte Männer mit 23,6 Prozent. Dies liegt an den selteneren Geldzahlungen für engagierte Frauen: Mit 5,8 Prozent erhalten Frauen im Rahmen ihrer freiwilligen Tätigkeit anteilig seltener Geldzahlungen als Männer mit 8,7 Prozent.

Bei der Betrachtung der Altersgruppen fallen vor allem die Unterschiede beim Erhalt der Sachzuwendungen für die freiwillige Tätigkeit auf (Abbildung 12-4a). Die Gruppe der 14- bis 29-Jährigen erhält von allen Altersgruppen mit 28,8 Prozent mit Abstand anteilig am häufigsten Sachzuwendungen. Auch Geldzahlungen und Sachzuwendungen in Kombination erhalten die Engagierten der jüngsten Altersgruppe anteilig deutlich häufiger als alle anderen Altersgruppen. 
Abbildung 12-4 Anteile freiwillig engagierter Personen, die Geldzahlungen und/oder Sachzuwendungen für die freiwillige Tätigkeit erhalten, 2019, a) gesamt, nach Geschlecht und nach Alter, b) nach Bildung (Basis: alle Engagierten)

a) gesamt, nach Geschlecht und nach Alter

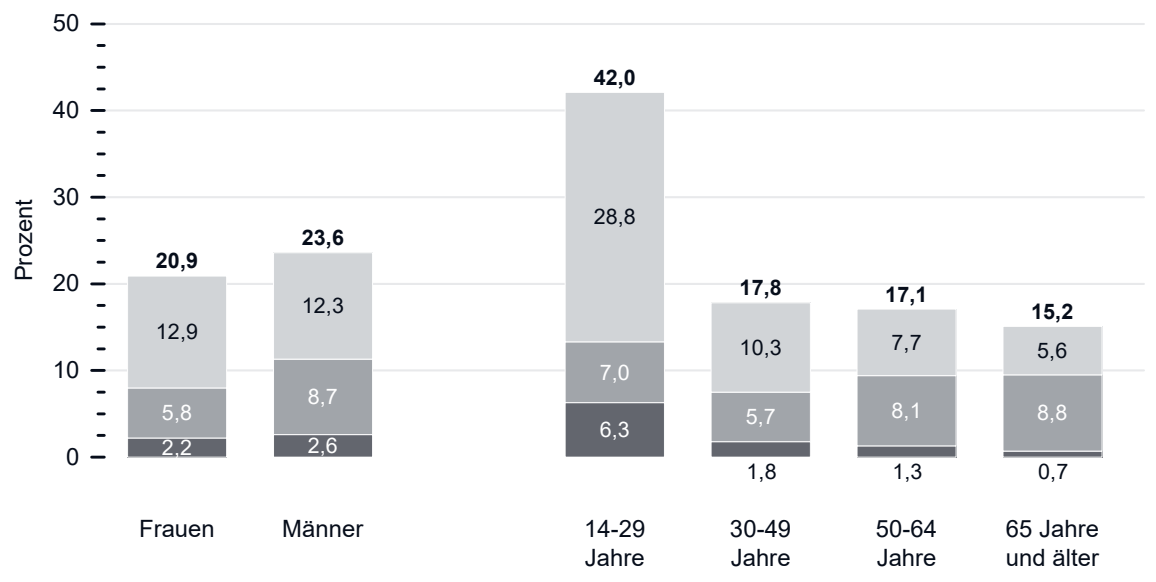

b) nach Bildung

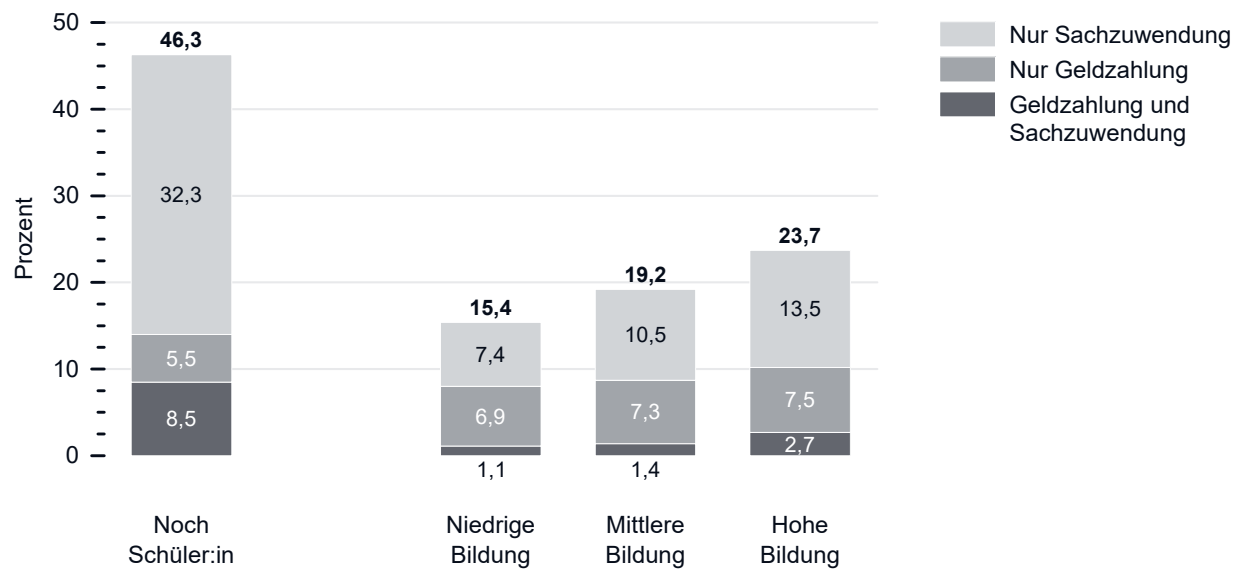

Quelle: FWS 2019, gewichtet, eigene Berechnungen (DZA). Basis: alle Engagierten ( $n=11.899$ ).

Hinweis: Abweichungen in den Prozentwerten ergeben sich durch das Auf- und Abrunden der Werte.

Für die Gesamtwerte aller Kategorien ist der Geschlechterunterschied statistisch signifikant; Altersgruppenunterschiede sind statistisch signifikant zwischen den 14- bis 29-Jährigen und allen anderen Altersgruppen; alle Bildungsgruppenunterschiede sind statistisch signifikant. Für die Kategorie,Nur Sachzuwendung' ist der Geschlechterunterschied nicht statistisch signifikant; alle Altersgruppenunterschiede sind statistisch signifikant; alle Bildungsgruppenunterschiede sind statistisch signifikant.

Für die Kategorie,Nur Geldzahlung' ist der Geschlechterunterschied statistisch signifikant; Altersgruppenunterschiede sind statistisch signifikant zwischen den 30- bis 49-Jährigen zu den beiden älteren Altersgruppen; Bildungsgruppenunterschiede sind nicht statistisch signifikant. Für die Kategorie,Geldzahlung und Sachzuwendung' ist der Geschlechterunterschied nicht statistisch signifikant; alle Altersgruppenunterschiede sind statistisch signifikant mit Ausnahme des Unterschieds zwischen den 30- bis 49-Jähriger und den 50- bis 64-Jährigen; alle Bildungsunterschiede sind statistisch signifikant mit Ausnahme des Unterschieds zwischen den Personen mit niedriger und mittlerer Bildung. 
Abbildung 12-5 Anteile der freiwillig Engagierten, die Geldzahlungen und/oder Sachzuwendungen für die freiwillige Tätigkeit erhalten, 2019, nach Bereichen (Basis: alle Engagierten)

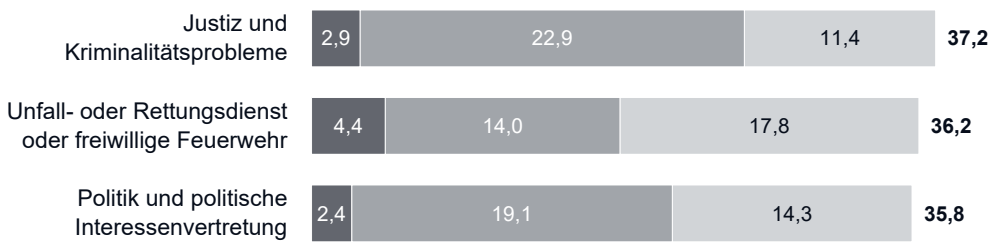

Außerschulische Jugendarbeit oder Bildungsarbeit für Erwachsene

Berufliche Interessenvertretung außerhalb des Betriebes

\begin{tabular}{|c|c|c|c|}
\hline 3,3 & 8,7 & 21,9 & 33 \\
\hline \multicolumn{4}{|l|}{1,1} \\
\hline & 10,5 & 20,6 & 32,2 \\
\hline
\end{tabular}

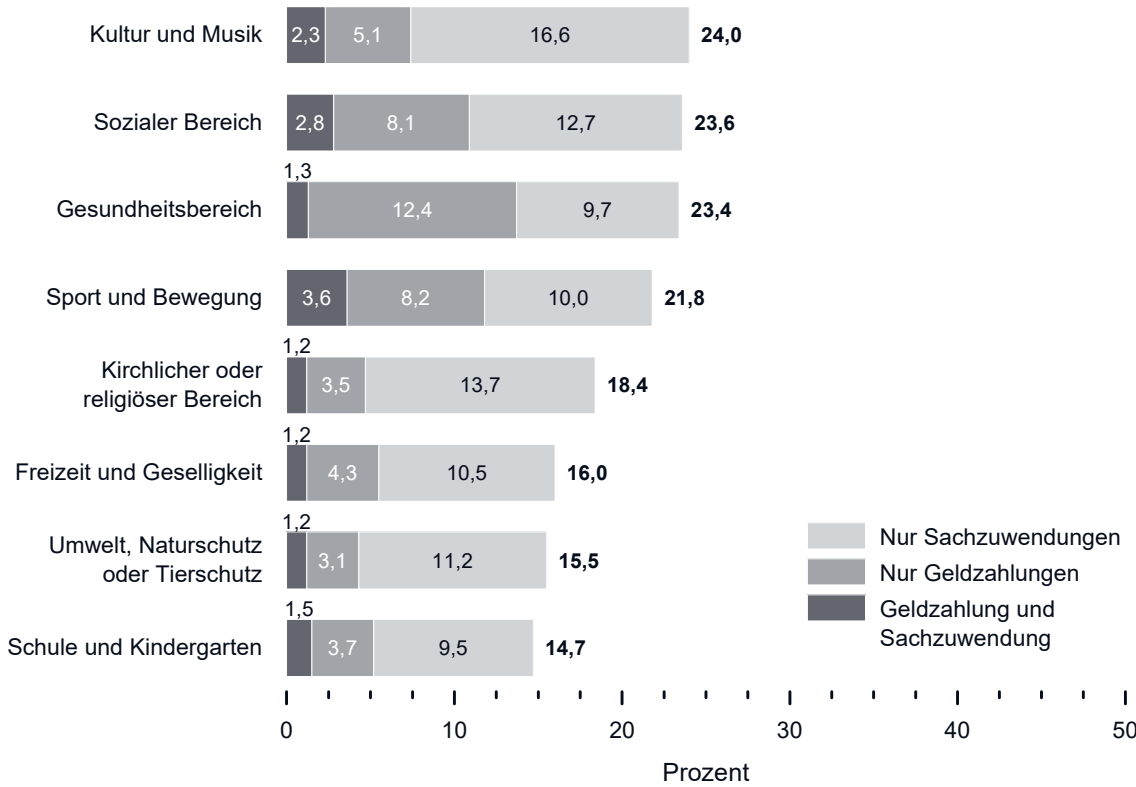

Quelle: FWS 2019, gewichtet, eigene Berechnungen (DZA). Basis: alle Engagierten ( $n=11.722$ ).

Hinweis: Abweichungen in den Prozentwerten ergeben sich durch das Auf- und Abrunden der Werte.

Für die Gesamtwerte aller Kategorien gibt es keine statistisch signifikanten Unterschiede zum Gesamtmittelwert aller Bereiche für die Bereiche Kultur und Musik, Sozialer Bereich, Gesundheitsbereich und Sport und Bewegung.

Für die Kategorie,Nur Sachzuwendung' gibt es keine statistisch signifikanten Unterschiede zum Gesamtmittelwert aller Bereiche für die Bereiche Justiz und Kriminalitätsprobleme, Politik und politische Interessenvertretung, Sozialer Bereich, Gesundheitsbereich, Kirchlicher oder religiöser Bereich, Freizeit und Geselligkeit sowie Umwelt, Naturschutz oder Tierschutz.

Für die Kategorie,Nur Geldzahlung' gibt es keine statistisch signifikanten Unterschiede zum Gesamtmittelwert aller Bereiche für die Bereiche Außerschulische Jugendarbeit oder Bildungsarbeit für Erwachsene, Berufliche Interessenvertretung außerhalb des Betriebes, Sozialer Bereich und Sport und Bewegung.

Für die Kategorie,Geldzahlung und Sachzuwendung' gibt es statistisch signifikante Unterschiede zum Gesamtmittelwert aller Bereiche für die Bereiche Sport und Bewegung und Freizeit und Geselligkeit. 
Im Altersgruppenvergleich erhalten die 30- bis 49-Jährigen mit 10,3 Prozent anteilig am zweithäufigsten Sachzuwendungen; die beiden älteren Gruppen der 50- bis 64-Jährigen und der 65-Jährigen und Älteren erhalten mit 7,7 Prozent beziehungsweise 5,6 Prozent anteilig am seltensten Sachzuwendungen. Hingegen erhalten die beiden ältesten Altersgruppen mit 8,1 Prozent und 8,8 Prozent anteilig am häufigsten Geldzahlungen im Rahmen ihrer freiwilligen Tätigkeit.

Engagierte, die noch zur Schule gehen, erhalten mit großem Abstand anteilig am häufigsten Sachzuwendungen mit 32,3 Prozent sowie Geld- und Sachzuwendungen in Kombination mit 8,5 Prozent für ihre freiwillige Tätigkeit (Abbildung 12-4b). Für die restlichen Bildungsgruppen ist zu beobachten, dass Engagierte mit hoher Bildung mit 13,5 Prozent anteilig häufiger Sachzuwendungen sowie mit 2,7 Prozent eine Kombination aus Geldzahlungen und Sachzuwendungen erhalten als Engagierte mit niedriger und mittlerer Bildung. Hinsichtlich der Geldzahlungen in der freiwilligen Tätigkeit gibt es zwischen den Bildungsgruppen keine statistisch signifikanten Unterschiede.

Auch für die gesellschaftlichen Engagementbereiche zeigen sich Unterschiede hinsichtlich der Geldzahlungen beziehungsweise Sachzuwendungen für die zeitaufwendigste oder einzige freiwillige Tätigkeit (Abbildung 12-5). Anteilig am häufigsten kommen mit über 30 Prozent Geldzahlungen sowie Sachzuwendungen in den Bereichen Justiz und Kriminalitätsprobleme (37,2 Prozent), Unfall- oder Rettungsdienst oder freiwillige Feuerwehr (36,2 Prozent), Politik und politische Interessensvertretung (35,8 Prozent), außerschulische Jugendarbeit oder Bildungsarbeit für Erwachsene (34,0 Prozent) und berufliche Interessensvertretung außerhalb des Betriebs (32,2 Prozent) vor. Hingegen kommen Geldzahlungen sowie Sachzuwendungen in den Bereichen Freizeit und Geselligkeit, Umwelt, Naturschutz oder Tierschutz sowie Schule und Kindergarten mit um die 15 Prozent anteilig besonders selten vor. Mit Fokus auf die Geldzahlungen für die freiwillige Tätigkeit zeigt sich, dass diese vor allem in den Bereichen Justiz und Kriminalitätsprobleme sowie Politik und politische Interessensvertretung mit über 19 Prozent anteilig überdurchschnittlich häufig vorkommen. Auch im Bereich Unfall- oder Rettungsdienst oder freiwillige Feuerwehr, im Gesundheitsbereich und bei der beruflichen Interessenvertretung außerhalb des Betriebes sind Geldzahlungen im Rahmen der freiwilligen Tätigkeit mit über 10 Prozent anteilig vergleichsweise häufig.

\subsection{Fazit}

Freiwillige Tätigkeiten sind der Definition nach nicht auf materiellen Gewinn ausgerichtet. Im freiwilligen Engagement gibt es jedoch die Möglichkeit der Kostenerstattungen für geleistete Auslagen sowie die Möglichkeit Sachzuwendungen und Geldzahlungen zu erhalten.

Insgesamt 44,o Prozent der Engagierten entstehen 2019 Kosten bei der Ausübung ihrer freiwilligen Tätigkeit. Dieser Anteil hat sich seit 2014 nicht verändert. Der Be- 
fund, dass für fast 6o Prozent der Engagierten, denen Kosten bei der Ausübung ihrer freiwilligen Tätigkeit entstehen, keine Möglichkeit der Kostenerstattung besteht verdeutlicht, dass ein erheblicher Anteil der Engagierten keine Möglichkeit hat, die Kosten erstattet zu bekommen. Weiterhin gibt es Unterschiede im Hinblick auf die Möglichkeit zur Kostenerstattung nach Geschlecht, nach Alter und nach Bildung. Für engagierte Männer ist die Möglichkeit der Kostenerstattung anteilig häufiger gegeben als für engagierte Frauen. Dies kann damit zusammenhängen, dass Frauen und Männer sich zum Teil in unterschiedlichen Kontexten engagieren. Es ist davon auszugehen, dass eine Kostenerstattung in Leitungs- und Vorstandspositionen im freiwilligen Engagement anteilig vergleichsweise häufig stattfindet; Frauen sind in diesen Positionen anteilig seltener vertreten als Männer (siehe Kapitel 9). Wenn die Möglichkeit der Kostenerstattung besteht, nutzen engagierte Frauen und Männer diese in gleichem Maße. Im Vergleich der Altersgruppen wird deutlich, dass vor allem die 14- bis 29-Jährigen anteilig am häufigsten von der Möglichkeit der Kostenerstattung im Rahmen ihrer freiwilligen Tätigkeit Gebrauch machen. Jüngere Engagierte erzielen häufig noch kein oder nur ein geringes eigenes Einkommen und sind vermutlich in stärkerem Maße auf die Kostenerstattung angewiesen als Engagierte aus höheren Altersgruppen. Die Möglichkeit der Kostenerstattung kann somit gerade für jüngere Engagierte eine wichtige Komponente für ihre Engagementbeteiligung darstellen. Weitere Unterschiede zeigen sich nach Bildungsstand: Für Engagierte mit hoher Bildung ist die Kostenerstattung anteilig häufiger möglich als für Engagierte mit niedriger oder mittlerer Bildung. Weiterhin machen Engagierte mit hoher Bildung von der Kostenerstattung anteilig häufiger Gebrauch als Engagierte mit niedriger oder mittlerer Bildung. Somit zeigt sich, dass höhergebildete engagierte Personen, mit einer in der Regel guten finanziellen Ressourcenausstattung, vergleichsweise häufig die Möglichkeit der Kostenerstattung nutzen können. Den Gruppen, die vermutlich in höherem Maße auf die Kostenerstattung angewiesen sind, steht diese Möglichkeit zu geringeren Anteilen offen.

Für Sachzuwendungen und Geldzahlungen im freiwilligen Engagement zeigt sich im Zeitvergleich, dass die Sachzuwendungen seit 1999 an Bedeutung gewonnen haben, wohingegen Geldzahlungen in den Jahren 2014 und 2019 im Vergleich zu den Vorjahren anteilig seltener sind. Dabei ist anzumerken, dass sich die Erfassung der Geldzahlungen über die Erhebungsjahre geändert hat. In den Jahren 2014 und 2019 wurden die Geldzahlungen separat von den Kostenerstattungen abgefragt. In den ersten drei Erhebungsjahren wurde hingegen unter anderem nach der ,pauschalierten Aufwandsentschädigung' gefragt, die keinen finanziellen Zugewinn bedeuten muss, sondern eine reine Kostenerstattung darstellen kann. Dadurch könnte die Bedeutung der Geldzahlungen in den ersten drei Erhebungsjahren überschätzt werden. Doch auch wenn wir die Kostenerstattung und Geldzahlungen kombiniert betrachten, bleibt der gezeichnete Trend über die Erhebungswellen hinweg weiterhin bestehen. Im Trendvergleich ist weiterhin zu erkennen, dass seit 2009 etwas mehr als ein Fünftel der engagierten Personen Geldzahlungen und/oder Sachzuwendungen 
erhalten. Im Umkehrschluss bedeutet das, dass die Mehrheit der Engagierten keine Geldzahlungen beziehungsweise Sachzuwendungen für ihre freiwillige Tätigkeit erhalten. Dieses Ergebnis kann einen wichtigen Rahmen für den Diskurs zu direkten Geldzahlungen im Engagement bilden, der in der Fachliteratur unter dem Stichwort ,Monetarisierung' geführt wird. Denn es zeigt sich: Es gibt keinen allgemeinen Trend zu vermehrten Geldzahlungen im freiwilligen Engagement.

Hinsichtlich der Sachzuwendungen und Geldzahlungen zeigen sich weiterhin Unterschiede zwischen verschiedenen Bevölkerungsgruppen. Engagierte Männer erhalten anteilig häufiger Geldzahlungen als engagierte Frauen. Ähnlich wie bei den Kostenerstattungen liegt es auch hier unter anderem daran, dass sich Frauen anteilig häufiger als Männer in Kontexten engagieren, in denen vermutlich öfter geringere Budgets für Geldzahlungen zur Verfügung stehen. Beispielsweise besetzen Frauen zu geringeren Anteilen Leitungs- und Vorstandspositionen im freiwilligen Engagement als Männer; Frauen sind zudem anteilig seltener in formell organisierten Vereinen und Verbänden und häufiger in individuell organisierten Gruppen engagiert als Männer (siehe Kapitel 9). Die jüngste Gruppe der 14- bis 29-jährigen Engagierten erhält von allen Altersgruppen anteilig am häufigsten Sachzuwendungen sowie Geldzahlungen und Sachzuwendungen in Kombination. Dies trifft auch auf die Engagierten $\mathrm{zu}$, die noch zur Schule gehen, die eine Teilmenge der Altersgruppe der 14- bis 29-jährigen Engagierten sind. Für die restlichen Bildungsgruppen zeigt sich, dass Engagierte mit hoher Bildung anteilig häufiger Geldzahlungen und/oder Sachzuwendungen erhalten als Engagierte mit niedriger und mittlerer Bildung. Ähnlich wie bei den Kostenerstattungen ist auch hier der Schluss zu ziehen, dass Engagierte mit einer meist guten Ressourcenausstattung zu höheren Anteilen Geldzahlungen sowie Sachzuwendungen erhalten können.

Die Analyse nach gesellschaftlichen Engagementbereichen zeigt, dass Geldzahlungen anteilig besonders häufig in den Bereichen Justiz und Kriminalitätsprobleme sowie Politik und politische Interessensvertretung getätigt werden. Durch die Tatsache, dass in diesen Bereichen engagierte Männer sowie Engagierte mit hoher Bildung zu höheren Anteilen vertreten sind als engagierte Frauen und Engagierte der anderen Bildungsgruppen, lässt sich ein Zusammenhang $\mathrm{zu}$ einigen vorherigen $\mathrm{Be}$ funden dieses Kapitels herstellen. Ein weiterer Bereich, in dem anteilig überdurchschnittlich häufig Geldzahlungen für die freiwillige Tätigkeit getätigt werden, ist der Gesundheitsbereich. In der Fachliteratur wird in Bezug auf den pflegerischen sowie schulischen Bereich problematisiert, dass die Finanz- und Personalknappheit durch den Einsatz von Engagierten, die Geldzahlungen erhalten, kompensiert werden könnte. Während die überdurchschnittlichen Geldzahlungen im Gesundheitsbereich für diese Beobachtung sprechen könnten, ist dies für den Bereich Schule und Kindergarten nicht der Fall; hier kommen die Geldzahlungen anteilig sogar besonders selten vor.

Zusammenfassend lässt sich sagen, dass einige - aber bei Weitem nicht alle - Engagierte Geldzahlungen und Sachzuwendungen im Rahmen ihrer freiwilligen Tätig- 
keit erhalten. Der Erhalt von Geldzahlungen und Sachzuwendungen im freiwilligen Engagement verteilt sich unterschiedlich auf die Bevölkerungsgruppen, wobei Bevölkerungsgruppen mit einer häufig ohnehin vorteilhaften Ressourcenausstattung diese Zahlungen und Zuwendungen zu höheren Anteilen erhalten als andere Bevölkerungsgruppen. Dies trifft auch auf die Möglichkeiten zur Kostenerstattung zu: Engagierte mit hoher Bildung haben zu höheren Anteilen Zugang zu Kostenerstattungen als Engagierte mit niedriger oder mittlerer Bildung. Mit Blick auf diesen Befund sollten die Organisationen und Einrichtungen den Zugang zu den Kostenerstattungen für Bevölkerungsgruppen mit einer geringeren Ressourcenausstattung verbessern.

\section{Literatur}

Beyer, T. (2017). Eigennutz statt Eigensinn? Betrachtungen zum aktuellen Stand der Debatte um eine Monetarisierung freiwilligen Engagements. In: Stiftung Mitarbeit (Hrsg.) Zwischen Erwerbsarbeit und Engagement. Die Debatte um das Geld im bürgerschaftlichen Engagement (S. 14-19). Bonn: Verlag Stiftung Mitarbeit.

Jakob, G. (2017). Wenn Engagement zu (Erwerbs-)Arbeit wird - Zu den Folgen einer Monetarisierung bürgerschaftlichen Engagements. In: Stiftung Mitarbeit (Hrsg.) Zwischen Erwerbsarbeit und Engagement. Die Debatte um das Geld im bürgerschaftlichen Engagement (S. 6-13). Bonn: Verlag Stiftung Mitarbeit.

Romeu Gordo, L., \& Vogel, C. (2017). Geldzahlungen, Sachzuwendungen und Kostenerstattung im freiwilligen Engagement. In: J. Simonson, C. Vogel \& C. Tesch-Römer (Hrsg.) Freiwilliges Engagement in Deutschland - Der Deutsche Freiwilligensurvey 2014 (S. 377-412). Wiesbaden: Springer VS.

Vandamme, R. (2018). Bürgerschaftliches Engagement und Teilhabe. In: E.-U. Huster, J. Boeckh \& H. Mogge-Grotjahn (Hrsg.) Handbuch Armut und soziale Ausgrenzung (S. 807-822). Wiesbaden: Springer VS.

Open Access Dieses Kapitel wird unter der Creative Commons Namensnennung Nicht kommerziell 4.0 International Lizenz (http://creativecommons.org/licenses/bync/4.0/deed.de) veröffentlicht, welche die nicht-kommerzielle Nutzung, Vervielfältigung, Bearbeitung, Verbreitung und Wiedergabe in jeglichem Medium und Format erlaubt, sofern Sie den/die ursprünglichen Autor(en) und die Quelle ordnungsgemäß nennen, einen Link zur Creative Commons Lizenz beifügen und angeben, ob Änderungen vorgenommen wurden.

Die in diesem Kapitel enthaltenen Bilder und sonstiges Drittmaterial unterliegen ebenfalls der genannten Creative Commons Lizenz, sofern sich aus der Abbildungslegende nichts anderes ergibt. Sofern das betreffende Material nicht unter der genannten Creative Commons Lizenz steht und die betreffende Handlung nicht nach gesetzlichen Vorschriften erlaubt ist, ist auch für die oben aufgeführten nicht-kommerziellen Weiterverwendungen des Materials die Einwilligung des jeweiligen Rechteinhabers einzuholen. 